Caso clínico

\title{
Saturnismo, a propósito de un caso
}

\author{
Lead poisoning. A case report
}

\section{Pablo Honorio Labanda Urbano ', Carmen Fernández García ${ }^{2}$}

1. Hospital de "La Princesa". Servicio de Prevención de Riesgos Laborales. Área 2. Madrid. España.

2. Inspección Médica. Instituto Nacional de la Seguridad Social. Dirección Provincial Madrid. España.

Recibido: 13-04-12

Aceptado: 07-05-12

\section{Correspondencia:}

Pablo Honorio Labanda Urbano

Hospital de La Princesa. S. ${ }^{\circ}$ Prevención Riesgos Laborales. Área 2.

Diego de León, 62

28006 Madrid. España.

Teléfono: (+34) 630831436

E-mail: pablohlabanda@gmail.com

\section{Resumen}

Profesora de pintura sobre vidrio y restauración de vidrieras de 65 años acude a la Unidad Médica de Valoración de Incapacidades del Instituto Nacional de la Seguridad Social (INSS) con diagnóstico de exposición laboral a plomo. La paciente ha permanecido en Incapacidad Temporal por astenia durante varios meses, y se le detecta tras una reincorporación al trabajo plumbemia elevada, asociada a otros síntomas típicos de la intoxicación por plomo, por lo que se la separa de su ambiente de trabajo.

Finalmente, el Equipo de Valoración de Incapacidades, reunido para valorar su caso resuelve Incapacidad Laboral Permanente Total derivada de enfermedad profesional para trabajos en que haya exposición a plomo y otros ototóxicos, exposición a ruidos de riesgo, y para aquellos trabajos en que sea necesaria una comunicación verbal fluida en frecuencias conversacionales normales.

Con este caso clínico pretendemos revisar los síntomas del cuadro clínico de saturnismo, así como hacer una reflexión sobre los efectos de la prevención de riesgos para el trabajador.

Med Segur Trab (Internet) 2012; 59 (227) 168-173

Palabras clave: intoxicación por plomo, pintura en vidrio, sistema nervioso, adultos.

Abstract

Lead poisoning is most commonly caused by occupational exposure. We report a case of a 65-year-old woman, paint teacher, which was working in reparation of stained glass window. She consults the Medical Incapacity Valoration Unit of National Institute of Social Security with diagnosis of lead occupational intoxication. The patient was not able to word for several months because of presenting asthenia. After reporting for work high lead blood levels were detected, in association with physical symptoms.

She was evaluated by a tribunal which settled total long sick-leave due to occupational lead exposure and was restricted for loud works and other ones that need verbal communication in conversational frequencies.

With this clinical case we try to check the symptoms of lead poisoning, as well as to think about the effects of prevention of occupational hazards.

Med Segur Trab (Internet) 2012; 59 (227) 168-173

Key words: lead poisoning, glass painting, nervous system, adults. 


\section{INTRODUCCIÓN}

El plomo se ha utilizado desde la antigüedad en la vida diaria de los hombres, gracias a sus particulares características que lo hacen especialmente dúctil y maleable. Entre los usos que se le han dado, destacan la fabricación de armamento, de material de consumo diario, así como su empleo en la construcción de edificios. Como consecuencia de esta utilización, la toxicidad por plomo es bien conocida desde la antigüedad. Existen referencias a dicha toxicidad en el antiguo Egipto (4000 a.C.). Hipócrates, en el siglo V a.C. describe el cólico saturnino como parte de la intoxicación por plomo, y en el primer compendio de Medicina del Trabajo, el tratado "de las Enfermedades de los Artesanos" Bernardino Ramazzini describe en el apartado de las enfermedades de los pintores un cuadro de temblor de manos y articulaciones, convulsiones, dolor intenso de estomago, caquexia, ennegrecimiento de los dientes y melancolía ${ }^{1}$.

En la actualidad el plomo constituye un contaminante no solo en el medio laboral, sino también en la vida diaria, ya que está presente en múltiples compuestos y lugares. Así, las acometidas de agua de muchos edificios antiguos son de plomo, que también se emplea como material de soldadura metálica. Además, derivados del plomo se emplean como antidetonantes en carburantes. De acuerdo con normativas internacionales, en los últimos 15 ó 20 años se está reduciendo la contaminación ambiental por plomo, principalmente por retirar las conducciones de agua hechas de plomo, y por disminuir el contenido de plomo de los carburantes. Estudios en este sentido refieren una disminución del plomo ambiental hasta de un $50 \%^{2}$.

\section{CASO CLÍNICO}

Trabajadora como profesora de pintura sobre vidrio y restauración de vidrieras desde 1996 permanece de baja laboral entre 2009 y 2010 durante 12 meses por un cuadro de astenia que limitaba sus actividades. Durante ese año es estudiada en servicio de Medicina Interna de un Hospital Terciario en relación con la astenia. Se detecta una lesión benigna tiroidea, por lo que en mayo de 2010 se le practica una tiroidectomía total. Tras la intervención persiste astenia que se relaciona con hipotiroidismo postquirúrgico, con hallazgos exploratorios y analíticos normales.

Previamente, en el mes de enero de 2010 se solicita por primera vez en sus analíticas una determinación de plumbemia, a raíz de una conversación casual con su médico sobre su actividad laboral. Se detecta plomo 12.8 (rango 0.1-10). Se diagnostica de intoxicación crónica por plomo, y se pauta tratamiento protocolizado, además de recomendarse evitar contacto con plomo. Esta plumbemia se determinó en el $8^{\circ}$ mes de baja laboral, por lo que llevaba 8 meses alejada del tóxico.

En mayo de 2010 (tras 13 meses de ausencia de exposición) la plumbemia era normal (8.1) En junio de 2010 es estudiada en el Hospital Clínico de Madrid por cuadro respiratorio consistente en disnea de moderados esfuerzos, tos desde hacia 5 años, con expectoración y ruidos torácicos. En este estudio se descarto neumoconiosis laboral.

En septiembre de 2010 retoma su actividad laboral, y a los pocos días comienza con un cuadro de cefalea, nauseas, vómitos, diarrea/estreñimiento y dolor abdominal cólico, así como poliartralgias, nicturia, depresión y astenia.

En octubre de 2010 (un mes después de la reincorporación) se realiza una nueva analítica, en la que se detecta una plumbemia de 71.2. En noviembre de 2010 la plumbemia era de 59.1. Se indica entonces interrupción de la exposición a plomo, por lo que causa baja laboral por su Mutua de Accidentes de Trabajo y Enfermedades Profesionales de la Seguridad Social el 15/11/2010, con una plumbemia de 52 (a fecha 26/11/2010).

En diciembre de 2010 se determina que la paciente presenta exposición de origen laboral a plomo, con un cuadro de exposición crónica a la que se sobreañade exposición 
aguda, con probable afectación cardiovascular, renal, osteoarticular (depósitos en corticales de huesos largos y calota detectados en una gammagrafía ósea realizada en abril de 2010) e intestinal (cólicos intestinales plúmbicos). Se indica cesión absoluta de la exposición.

La paciente queda con tratamiento, debiendo seguir las recomendaciones del servicio de Nefrología. Se remite a control por su Médico de Atención Primaria, que en la actualidad coordina la atención especializada (a cargo de Medicina Interna y Nefrología).

Como antecedentes personales, presenta de forma aguda un cólico renal en 2004, crisis de gota en 2005, bronquitis, depresión en 2007 y neumonía en 2008.

En cuanto a la sintomatología crónica la paciente sufría cefaleas, nauseas, vómitos, episodios de diarrea alternados con estreñimiento, dolor abdominal cólico, poliartralgias, nicturia, depresión, dificultad respiratoria, disnea, y astenia severa.

La trabajadora acude a Inspección Médica de la Seguridad Social refiriendo la sintomatología antes mencionada, así como pérdida de audición de varios años, y aporta los informes que obran en su poder. Se solicita valoración por la consulta de Otorrinolaringología de la Inspección, para realización de estudio audiométrico que complete el caso. Los resultados de dicha audiometría concluyen hipoacusia neurosensorial simétrica, con UMC en ambos oídos a $70 \mathrm{~dB}$. En audiometría verbal tiene discriminación de $100 \%$ a $75-85$ dB. Romberg negativo, Rinne positivo bilateral.

La paciente refiere en la consulta de la Unidad de Valoración de Incapacidades del INSS que en su trabajo no ha habido reconocimientos medico-laborales ni iniciales ni periódicos en función de la exposición a tóxicos. Además presenta documentación referente a unas propuestas de medidas correctoras emitidas por el Servicio de Prevención Ajeno en 2002 a instancia de la trabajadora. Aporta también una nueva valoración por el mismo Servicio en 2010, en el que queda reflejado que no se han aplicado dichas medidas correctoras. Estas se centraban principalmente en dos aspectos relevantes del medio de trabajo, que eran la ausencia de extracción localizada de aire y la ausencia de vestuarios con armarios diferenciados para ropa de trabajo y de calle, así como de duchas.

Finalmente, el Equipo de Valoración de Incapacidades del Instituto Nacional de la Seguridad Social, reunido para valorar su caso resuelve Incapacidad Permanente Total derivada de enfermedad profesional para trabajos en que haya exposición a plomo y otros ototóxicos, exposición a ruidos de riesgo, y para aquellos trabajos en que sea necesaria una comunicación verbal fluida.

\section{EXPOSICIÓN DEL CASO}

\section{Valores límite y conceptos relativos a la exposición al plomo}

Según el protocolo de vigilancia sanitaria específica para plomo del Ministerio de Sanidad, se considera como trabajador expuesto a aquel que durante mas de 30 días al año se encuentra con concentración ambiental $>40 \mathrm{mg} / \mathrm{m}^{3}$ de aire, referido a 8 horas diarias y 40 semanales, o aquel que presenta plumbemias mayores de $40 \mathrm{ug} / 100 \mathrm{ml}$ para hombres o mayores de $30 \mathrm{ug} / 100 \mathrm{ml}$ para mujeres ${ }^{3}$.

Se consideran niveles de acción para el plomo (aquellos desde los que se debe adoptar vigilancia biológica de los trabajadores) una concentración ambiental de $75 \mathrm{mg} / \mathrm{m}^{3}$, referido a 8 horas diarias y 40 semanales o una plumbemia de $40 \mathrm{ug} / 100 \mathrm{ml}$.

Los Valores Límite de Exposición son aquellos que no deben ser superados, debiendo alejarse al trabajador de la exposición en caso de rebasarlos. Para el plomo son $150 \mathrm{mg} / \mathrm{m}^{3}$ de aire referido a 8 horas diarias y 40 semanales o plumbemias mayores de $70 \mathrm{ug} / 100 \mathrm{ml}^{2,3}$. 
En el listado de actividades con exposición a plomo, dentro del subgrupo de elevado riesgo se encuentran, entre otras, la soldadura de objetos y aleaciones de plomo, y la fabricación y utilización de pinturas, esmaltes y barnices compuestos de sales y óxidos de plomo $^{3}$.

\section{Dinámica del plomo en el organismo}

La vía de penetración más importante es la respiratoria, y su grado de absorción depende de la concentración ambiental, del tiempo de exposición y de la forma física y química del producto, así como de las condiciones de trabajo y de factores intrínsecos del trabajador. Más del 50\% del plomo aspirado pasa a sangre tras 50 horas de exposición.

La segunda vía de absorción en importancia es la oral. Entre un 5 y un $10 \%$ del plomo ingerido pasa a sangre, eliminándose el resto por las heces. Por último, la vía cutánea, que es muy débil en el caso del plomo.

La eliminación del plomo es por vía urinaria principalmente. Por vía fecal, se elimina el $80 \%$ del plomo ingerido más una pequeña parte del plomo absorbido que se elimina por la bilis. Otras vías son la saliva, el sudor, o la leche ${ }^{3}$.

\section{Efectos del plomo en el organismo}

En el tejido hematopoyético, el plomo altera la maduración de los glóbulos rojos por inhibición de la síntesis del grupo Hem en los eritroblastos. De esta manera se generan megaloblastos y eritroblastos poliploides que presentan el punteado basófilo que es característico de esta intoxicación. Además degrada el ARN de los reticulocitos en vías de maduración. Respecto a la sangre circulante, aparece una anemia saturnina, que es hemolítica y megaloblástica, con glóbulos rojos más grandes, más frágiles y con vida media disminuida ${ }^{4}$.

En el sistema nervioso, el plomo interfiere en el metabolismo de la acetilcolina, enlenteciendo el impulso nervioso. Este hecho aparece con plumbemias superiores a $60 \mathrm{ug} / 100 \mathrm{ml}^{4}$.

La afectación del sistema renal es progresiva. En una primera fase aparecen inclusiones intranucleares de plomo en las células tubulares, sin afectar la función renal. A continuación aparece fibrosis intersticial, y comienza la afectación funcional. Por último aparece una nefritis crónica, con lesión tubular y glomerular irreversible ${ }^{2,4}$.

Sobre la reproducción, el plomo aumenta la tasa de morbi-mortalidad perinatal, la hipospermia y la tasa de abortos espontáneos. El plomo pasa la barrera hematoplacentaria hasta igualar a ambos lados la plumbemia ${ }^{4}$.

En el tejido óseo desplaza el calcio de los huesos, para depositarse en ellos, con lo que aumenta la fragilidad de los mismos.

El plomo produce hipertensión y aumenta el riesgo cardiovascular por afectación renal y por aumento de la resistencia vascular. Además infiltra el tejido de conducción cardiaca pudiendo producir alteraciones del ritmo cardiaco ${ }^{4}$.

\section{Clínica}

La intoxicación aguda por plomo (infrecuente en la actualidad) asocia dolor abdominal importante, vómitos, estreñimiento, convulsiones y estado de coma que puede llevar a muerte, delirios, episodios psicóticos, oliguria y citolisis en los hepatocitos que puede llevar a necrosis hepática.

La intoxicación crónica, más frecuente en la actualidad, diferencia tres fases. En una primera fase de impregnación, con plumbemias menores de $70 \mathrm{ug} / 100 \mathrm{ml}$ aparece sintomatología imprecisa, estreñimiento, mal estar general, modificaciones de humor, fatiga, artromialgias, insomnio, disminución de la velocidad de impulso nervioso en extremidades, y comienzan a evidenciarse alteraciones del tejido hematopoyético. En la 
siguiente fase, de franca intoxicación, aparece el cólico saturnino, polineuritis motoras que afectan más a los músculos más activos, hipoacusia neurosensorial simétrica, encefalopatía saturnina (afasia transitoria, hemianopsias, discromatopsia, disminución de la capacidad intelectual...), disminución de la captación tiroidea de yodo e hipospermia. Finalmente, la fase terminal de la intoxicación crónica presenta hipertensión permanente y nefritis asociada a gota y a alteraciones del ritmo cardiaco, además de los síntomas y signos de la fase anterior ${ }^{2,3,4}$.

\section{Tratamiento}

El tratamiento consiste en el alejamiento de la fuente de plomo, y la administración de suplementos cálcicos a dosis de prevención de osteoporosis. Además, en los casos de plumbemias elevadas $(60 \mathrm{ug} / 100 \mathrm{ml}$ ) o gran afectación clínica esta indicada la quelación. Actualmente se recomienda como quelante el ácido dimercaptosuccínico (DMSA), ya que tiene pocos efectos adversos, y no redistribuye el plomo al cerebro. Otras opciones son el edetato disódico cálcico (EDTA Ca), y la asociación de este con dimercaprol en los casos en que hay encefalopatía ${ }^{2,3,4}$.

\section{DISCUSIÓN Y CONCLUSIONES}

Desde el punto de vista de la vigilancia de la salud, es necesario ofrecer a los trabajadores formación e información sobre los riesgos para la salud que entraña el plomo, sobre las medidas higiénicas a adoptar ante el mismo, sobre los peligros de fumar, beber y comer en los puestos de trabajo (que deberá estar prohibido) y sobre la utilidad y obligatoriedad de la ropa de trabajo y los EPIs, así como su conservación ${ }^{3,5}$.

En la actualidad, diferentes normativas de carácter internacional están consiguiendo que se reduzcan los niveles de plomo ambiental hasta un 50\%. Esto se ha conseguido en parte gracias a la disminución del plomo en los carburantes, a la retirada de tuberías de plomo en edificios antiguos, al empleo de nuevos compuestos para estas instalaciones en edificios de nueva construcción, o a la retirada del plomo en los productos de pintura.

Aun así sigue habiendo determinadas actividades en las que no se ha podido retirar este compuesto, debido a las especiales características de determinadas aplicaciones, como puede ser, en este caso, la fijación de pintura translúcida a vidrios que quedan a la intemperie.

Según la Ley de PRL, entre los principios generales de la actuación preventiva se encuentra la implantación de métodos de protección colectiva e individual para aquellos riesgos que no son evitables, anteponiendo siempre la protección colectiva. Además, de acuerdo con la Guía Técnica de agentes químicos, cuando la naturaleza de la actividad no permita la eliminación del riesgo por sustitución, el empresario garantizará la reducción al mínimo de dicho riesgo aplicando medidas de prevención y protección que sean coherentes con la evaluación de riesgos ${ }^{6}$.

En este caso clínico hacemos mención a unas medidas correctoras que emitió el Servicio de Prevención Ajeno a petición de la trabajadora, así como a un informe emitido por el mismo Servicio ocho años después, en el que refleja que no se han aplicado dichas medidas.

Es necesario reflexionar sobre el papel que juega la prevención de riesgos laborales en la vida de los trabajadores, y las consecuencias que pueden derivarse de la omisión de las medidas que se proponen desde los Servicios de Prevención después de una evaluación de riesgos. En este caso concreto queda patente que una prevención eficaz, con un respaldo adecuado por parte de la empresa puede evitar complicaciones posteriores que afectan tanto a los trabajadores como a los empresarios.

Por otra parte, hemos visto que en los controles de plumbemia que se han realizado a la trabajadora tan solo uno de ellos supera, y lo hace escasamente, los límites de 
exposición admitidos por el Instituto Nacional de Seguridad e Higiene en el Trabajo. Aún así, la clínica apunta claramente a un cuadro de saturnismo. En la ACGIH (American Conference of Governmental Industrial Hygienists) el valor límite que se propone es de $30 \mu \mathrm{g} / 100$, que coincide con el que implanta la OSHA (Occupational Safety and Health Administration). ${ }^{2}$ Con esta idea queremos incidir en que quizá seria recomendable revisar los valores límite de exposición que se consideran válidos, ya que quizá se están pasando por alto casos de exposición laboral a plomo.

\section{REFERENCIAS BIBLIOGRÁFICAS}

1. Ramazzini B. Tratado de las Enfermedades de los Artesanos. Escuela Nacional de Medicina del TrabajoInstituto de Salud Carlos III. Madrid, 2007. Capítulo IX, de las enfermedades de los pintores. Págs. 119-120.

2. Plomo. Toxicología. Disponible en la página Web de la Universidad de Zaragoza: http://wzar.unizar.es/ stc/toxicologianet/pages/x/x17/x17f/09.htm. Consultada el 10/03/2012.

3. Padilla Magunazelai A, Rodríguez-Sierra Huguet N, Martínez Castillo A Protocolo de vigilancia sanitaria especifica de Plomo. Ministerio de Sanidad y Consumo. Madrid, 1999.

4. Brawnwald E, Hauser S, Fauci A, Longo D, Kasper D, Jameson JL. Harrison, Principios de Medicina Interna. 15 Edición, Mc Graw-Hill, Madrid 2002. Intoxicación por Metales Pesados, sección específica de Plomo. Págs. 3034-3035.

5. Real Decreto 374/2001, de 6 de abril, sobre la protección de la salud y seguridad de los trabajadores contra los riesgos relacionados con los agentes químicos durante el trabajo. Disponible en la pagina web del BOE: http://www.boe.es/aeboe/consultas/bases_datos/doc.php?id=BOE-A-2001-8436

6. Ley 31/1995, de 8 de noviembre, de Prevención de Riesgos Laborales. Editada en el BOE n. ${ }^{\circ}$ 269, de 10/11/1995. Disponible en la pagina web del BOE: http://www.boe.es/aeboe/consultas/bases_datos/doc. php?id=BOE-A-1995-24292.

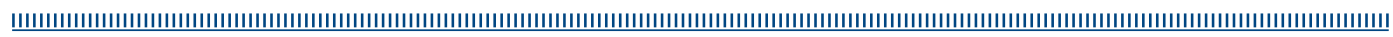

